\title{
To the Issue of Reconciling Quantum Mechanics and General Relativity
}

\author{
Fisenko S. I \\ Rusthermosynthesis JSC, Moscow, Russia, Stanislav
}

\begin{abstract}
The notion of gravitational radiation as a radiation of the same level as the electromagnetic radiation is based on theoretically proved and experimentally confirmed fact of existence of stationary states of an electron in its gravitational field characterized by the gravitational constant $K=10^{42} G(G$ is the Newtonian gravitational constant) and unrecoverable space-time curvature $\Lambda$. If the numerical values of $K \approx 5.1 \times 10^{31}$ $\mathrm{Nm}^{2} \mathrm{~kg}^{-2}$ and $\Lambda=4.4 \times 10^{29} \mathrm{~m}^{-2}$, there is a spectrum of stationary states of the electron in its own gravitational field (0.511 MeV ... $0.681 \mathrm{MeV})$.Adjusting according to the known mechanisms of broadening does not disclose the broadening of the registered portion of the emission spectrum of the micropinch. It indicates the presence of an additional mechanism of broadening the registered portion of the spectrum of the characteristic radiation due to the contribution of the excited states of electrons in their own gravitational field. The energy spectrum of the electron in its own gravitational field and the energy spectra of multielectron atoms are such that there is a resonance of these spectra. As obvious, the consequence of such resonant interaction is appearance, including new lines, of electromagnetic transitions not associated with atomic transitions. The manuscript is the review of previously published papers cited in the references.
\end{abstract}

\section{Introduction}

Generally covariant relativistic form of the equations of Einstein's theory of gravitation, as we know, has the following form:

$R_{i k}-\frac{1}{2} g_{i k} R-\Lambda g_{i k}=\chi T_{i k}$

In these equations $\chi$ is a constant that relates to the geometrical properties of space-time distribution of physical matter, so that the origin of the equations is not associated with the numerical restriction of the values. Only the requirements of compliance with the Newton's law of universal gravitation leads to numerical values $\Lambda=0, \chi=8 \pi G / c^{4}$, where $\mathrm{G}$ is the Newton's gravitational constant. Equation with certain constants determined in such a way and so are the equations of the Einstein's general relativity theory. The equations (1) are a common mathematical form of the gravitational field equations corresponding to the principle of equivalence and the postulate of general covariance. The equations of the form (1) are obtained simultaneously by Einstein and independently by Gilbert [1]. At the same time, we refer to A. Salam [2], as he is the one of the first who drew attention to the discrepancy between the quantum level of the numerical value of the Newtonian gravitational constant. It is he who proposed the concept of "strong" gravity, which was based on the assumption of the existence of f-mesons of spin-2 generating SU (3) multiplet (described by Pauli-Fierz equation). It was shown that the possibility of another link constant along with the Newtonian is not inconsistent with the observed effects [2]. This approach was not further developed due to a number of reasons. It is clear now that the numerical value of the "strong" gravity constant should be used in equations (1) with $\Lambda \neq 0$. By the way, when $\Lambda \neq 0$ there are stationary solutions of general Einstein's equations as it was stated by Einstein himself, but after the discovery by A. Friedman [3] non-stationary solutions when $\Lambda=0$, finally the general theory of relativity was formed as it is now known. The decisive argument in general relativity for equating $\Lambda$ member to zero is the need to correct limiting transition to Newton's theory of gravitation.

Starting from the 70s it became evident [2] that in the quantum domain numerical value of $\mathrm{G}$ is not compatible with the principles of quantum mechanics. A number of studies [2] have shown that in the quantum domain acceptable is constant $K$, wherein $K \approx 10^{40} G$. This marked the problem of generalization of the relativistic equations for the quantum level: this generalization should join the numerical values of the constants in quantum and classical fields.

2.Gravitational radiation of the electrons with line spectrum as being of one level of the electromagnetic radiation.

In the development of these results as an approximation to the micro level of the field equations of Einstein, a model based on the following assumptions is proposed: 
The gravitational field in the region of localization of elementary particles with mass $m_{0}$ is characterized by the values of the gravitational constant $K$ and constant $\Lambda$, which lead to the stationary states of a particle in its own gravitational field, and stationary states of the particles themselves are the source of the gravitational field with the Newtonian gravitational constant $G$.

The complexity of solving this problem forced to turn to the simplest approximation, namely the calculation of the energy spectrum only in the approximation of the fine structure due to relativism. In this approximation, the problem for the stationary states of an elementary source in its own gravitational field is reduced to the solution of the following equations:

$$
\begin{aligned}
& f^{\prime \prime}+\left(\frac{v^{\prime}-\lambda^{\prime}}{2}+\frac{2}{r}\right) f^{\prime}+e^{\lambda}\left(K_{n}^{2} e^{-v}-K_{0}^{2}-\frac{l(l+1)}{r^{2}}\right) f=0 \\
& -e^{-\lambda}\left(\frac{1}{r^{2}}-\frac{\lambda^{\prime}}{r}\right)+\frac{1}{r^{2}}+\Lambda=\beta(2 l+1)\left\{f^{2}\left[e^{-\lambda} K_{n}^{2}+K_{0}^{2}+\frac{l(l+1)}{r^{2}}\right]+f^{\prime 2} e^{-\lambda}\right\} \\
& -e^{-\lambda}\left(\frac{1}{r^{2}}+\frac{v^{\prime}}{r}\right)+\frac{1}{r^{2}}+\Lambda=\beta(2 l+1)\left\{f^{2}\left[K_{0}^{2}-K_{n}^{2} e^{-v}+\frac{l(l+1)}{r^{2}}\right]-e^{\lambda} f^{\prime 2}\right\} \\
& \left\{-\frac{1}{2}\left(v^{\prime \prime}+v^{\prime 2}\right)-\left(v^{\prime}+\lambda^{\prime}\right)\left(\frac{v^{\prime}}{4}+\frac{1}{r}\right)+\frac{1}{r^{2}}\left(1+e^{\lambda}\right)\right\}_{r=r_{n}}=0
\end{aligned}
$$

(5)

$$
\begin{aligned}
& f\left(\sqrt{\Lambda^{-1}}\right)=0 \\
& f\left(r_{n}\right)=0
\end{aligned}
$$

(7)

$$
\begin{aligned}
& R\left(\sqrt{\Lambda^{-1}}\right)=\Lambda \\
& \int_{\sqrt{\Lambda^{-1}}}^{r_{n}} f^{2} r^{2} d r=1
\end{aligned}
$$

(9)

Equations (2)—(4) follow from equations (10)—(11)

$$
\begin{aligned}
& \left\{-g^{\mu \nu} \frac{\partial}{\partial x_{\mu}} \frac{\partial}{\partial x_{v}}+g^{\mu v} \Gamma_{\mu \nu}^{\alpha} \frac{\partial}{\partial x_{\alpha}}-K_{0}^{2}\right\} \Psi=0 \\
& R_{\mu v}-\frac{1}{2} g_{\mu v} R=-\kappa\left(T_{\mu v}-\mu g_{\mu v}\right)
\end{aligned}
$$

After the substitution of $\Psi$ in the form of $\Psi=f_{E l}(r) Y_{l m}(\theta, \varphi) \exp \left(\frac{-i E t}{\hbar}\right)$ into them and specific computations in the central-symmetry field metric with the interval defined by the expression [6]

$$
d S^{2}=c^{2} e^{v} d t^{2}-r^{2}\left(d \theta^{2}+\sin ^{2} \theta d \varphi^{2}\right)-e^{\lambda} d r^{2}
$$

Above indicated: $f_{E l}$ is radial wave function describing the state with a definite energy $\mathrm{E}$ and the orbital angular momentum $l$ (hereinafter indexes $E l$ are omitted), $Y_{l m}(\theta, \varphi)$ are spherical functions, $K_{n}=E_{n} / \hbar c$, $K_{0}=c m_{0} / \hbar, \beta=(\kappa / 4 \pi)\left(\hbar / m_{0}\right) \quad \kappa=8 \pi K / c^{4}$.

Right-hand sides of equations (3)-(4) are calculated from the general expression for the energy-momentum tensor of a complex scalar field:

$$
T_{\mu \nu}=\Psi_{, \mu}^{+} \Psi_{, v}+\Psi_{, \nu}^{+} \Psi_{, \mu}-\left(\Psi_{, \mu}^{+} \Psi^{, \mu}-K_{0}^{2} \Psi^{+} \Psi\right) g_{\mu \nu}
$$

The respective components $T_{\mu \nu}$ are obtained summing by index $m$ using specific identities for the spherical functions [5] after the substitution in (13) $\Psi=f(r) Y_{l m}(\theta, \varphi) \exp \left(\frac{-i E t}{\hbar}\right)$. 
In the simplest (in terms of initial mathematical evaluations) approximation, the problem for stationary states in its gravitational field (with the constants $K$ and $\Lambda$ ) was solved in [6]. From the solution of this problem should be:

a) If the numerical values of $K \approx 5.1 \times 10^{31} \mathrm{Nm}^{2} \mathrm{~kg}^{-2}$ and $\Lambda=4.4 \times 10^{29} \mathrm{~m}^{-2}$, there is a spectrum of stationary states of the electron in its own gravitational field $(0.511 \mathrm{MeV} \ldots 0.681 \mathrm{MeV})$. The main state is the observed electron rest energy $0.511 \mathrm{MeV}$. This numerical value of $\Lambda$ has important physical meaning: an introduction to the Lagrangian density permanent member, not dependent on the state of the field. This implies the existence of unremovable space-time curvature, not affiliated with any matter, nor with the gravitational field.

b) These steady states are the sources of the gravitational field with constant $G$.

c) Transitions between the stationary states of the electron in its own gravitational field leads to gravitational radiation, which is characterized by a constant $K$, that gravitational radiation is the emission of the same level as electromagnetic (electric charge e, gravitational charge ) $m \sqrt{K}$. In this regard, it makes no sense to say that the gravitational effects in the quantum region are characterized by the constant $G$. This constant applies only to the macroscopic field and it can not be transferred to the quantum level (which, by the way, we remind, show negative results for the detection of gravitational waves with the constant G, and they can not be). It is considered that according to General Relativity (GR), gravitational radiation can generate only system with variable quadrupole or higher multipole moments. Under this assumption, the corresponding power of gravitational radiation is determined by the relationship:

$L=\frac{1}{5} \frac{G}{c^{5}}\left\langle\frac{d^{3} Q_{i j}}{d t^{3}} \frac{d^{3} Q^{i j}}{d t^{3}}\right\rangle$,

where $Q_{i j}$ is a quadrupole moment tensor of the mass distribution of the radiating system, and the constant in this relationship defines the order of magnitude of the radiation power. Wrongfulness of this formula, as follows from the above, is not to use the quadrupole approximation, but in the scheme calculation. The presence of stationary states in the own gravitational field allows the correct calculation of gravitational radiation in the strict quantum approach based on the spectrum of transitions to stationary states already with constant $\mathrm{K}$. It is permissible to use not only quadrupole, but also dipole quantum approach, which is quite obvious. Gravitational waves with the constant $G$ do not exist. That is evidenced by the negative results of the detection of gravitational waves, based on the assumption of a completely illegitimate assumption of gravitational wave generation by any mass distribution with variable multipole (starting with quadrupole) moments.

The presence of the stationary states of the electron in its own gravitational field is in full compliance with the special theory of relativity. According to STR, relativistic relation between energy and momentum is broken, if we assume that the total energy of the electron is determined only by the Lorentz electromagnetic energy [7]. In more detail the situation is as follows [7]. The energy and momentum of a moving electron (assuming that the distribution of electric charge is spherically symmetric) is determined by:

$$
\begin{aligned}
\bar{P} & =\bar{V} \frac{\frac{4}{3} E_{0} / c^{2}}{\sqrt{1-\beta^{2}}} \\
E & =\frac{E_{0}\left(1+\frac{1}{3} V^{2} / c^{2}\right)}{\sqrt{1-\beta^{2}}}
\end{aligned}
$$

If this equations determined both the total momentum and total energy, there would have been the equation:

$$
E=\int\left(\bar{V} \frac{d \bar{P}}{d t}\right) d t
$$

However this relation does not exist because the integral in the right part is equal to:

$$
\frac{\frac{4}{3} E_{0}}{\sqrt{1-\beta^{2}}}+\text { const }
$$


If to assume that the momentum contrary to the energy has purely electromagnetic character, the total energy of the moving electron and the total energy of resting electron as well as for the rest mass, we would have the relations:

$E^{\prime}=\frac{E_{0}^{\prime}}{\sqrt{1-\beta^{2}}}, E_{0}^{\prime}=\frac{4}{3} E_{0}, m_{0}=\frac{E_{0}^{\prime}}{c^{2}}=\frac{4}{3} \frac{E_{0}}{c^{2}}$,

where the rest mass is determined by the equation:

$\bar{P}=\frac{m_{0} \bar{V}}{\sqrt{1-\beta^{2}}}$

Then (18) implies that the total energy of an electron at rest is $4 / 3$ of its Lorentz electromagnetic energy. That corresponds to the numerical data on the spectrum of stationary states of the electron in its own gravitational field. In the Standard Model the relativistic relation between the energy and momentum of the electron is broken, as it is assumed that the total energy of the electron is determined only by the Lorentz electromagnetic energy. This follows from the fact that the gravitational interaction at the quantum level in the Standard Model is not considered.

It should immediately be noted that the numerical evaluation of the spectrum is approximate. The greatest uncertainty is the estimation of the numerical value of the first steady state, as more and more accurate as you approach $E_{\infty}=171 \mathrm{keV}$.

With the help of the Kerr-Newman metric evaluation of the numerical values of K [8] can be obtained by the formula:

$$
K=\frac{r^{2}}{\left(m c r^{2} / L-L / m c\right)\left(m / r c^{2}-e^{2} / r^{2} c^{4}\right)}
$$

where $\mathrm{r}, \mathrm{m}, \mathrm{e}, \mathrm{L}, \mathrm{c}$ are the classical electron radius, mass, charge, orbital angular momentum, the speed of light, correspondingly. The numerical value of the orbital angular momentum was taken equal to the spin of the electron.Thus, we can assume that the physical nature of spin is possible that this value is the orbital angular momentum of the particles in their own gravitational field. This gives ground to consider that the use of KleinGordon equation is not so easy. The distance at which the gravitational field with the constant $\mathrm{K}$ is localized is less than the Compton wavelength, and for the electron, for example, this value is of the order of its classical radius. At distances larger than this one, the gravitational field is characterized by the constant G, i.e., correct transition to Classical GR holds.

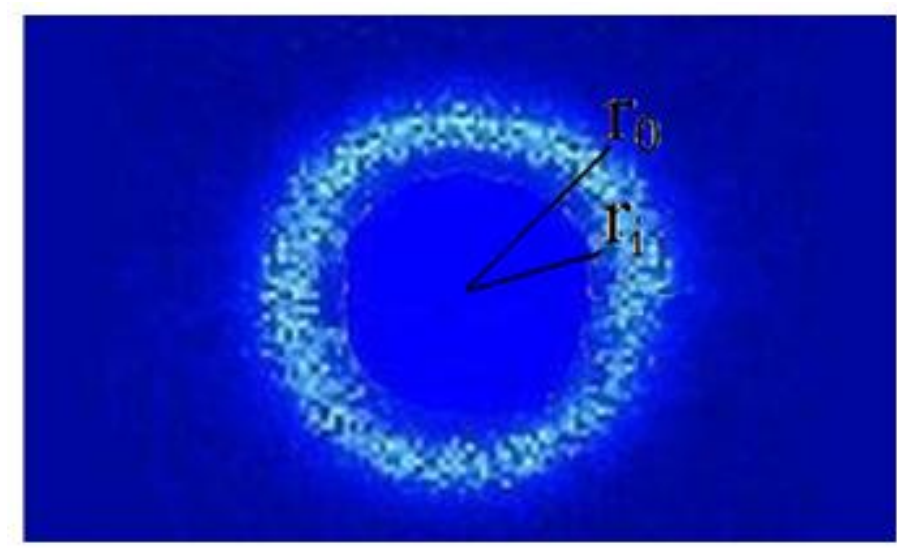

Figure 1.Electron in proper gravitational field in the ground stationary state.

$\mathrm{r}_{\mathrm{i}}$ - radius of irremovable curvature,

$\mathrm{r}_{0}$ - "classical" electron radius being a radius of the ground stationary state of electron in proper gravitational field

There is a problem with the measurement of the proton's size.There are several approaches. One is relating to the elastic scattering of electrons on protons. The second uses a high-precision laser spectroscopy. The matter is that levels in the atom depend on the final size of nucleus. If we work with hydrogen, then the 
nucleus is the proton. Thus, different methods produce results, inconsistent on 7 sigma. These results are stated in [20]. And there is no explanation generally accepted. At the same time, on a qualitative level, the existence of stationary states of a proton in its own gravitational field would explain these results. In the process of measuring the transitions on these stationary states occur (including the possible resonance of the said spectra of electron and proton), corresponding to the different states of a proton, which leads to different results. That is, it is not the measurement accuracy and in that the different states of the proton are measured depending on the measurement method. It is very important (along with the broadening of the emission spectra of high temperature plasma of multiply charged ions) result for the quantitative registration of the particles spectra in its own gravitational field.

\section{The spectra of the characteristic radiation of stars and laboratory plasmas}

Figure 2 shows the characteristic parts of the spectrum of soft $\mathrm{x}$-rays micropinches. Adjusting according to the known mechanisms of broadening does not disclose the broadening of the registered portion of the emission spectrum of the micropinch. It indicates the presence of an additional mechanism of broadening the registered portion of the spectrum of the characteristic radiation due to the contribution of the excited states of electrons in their own gravitational field
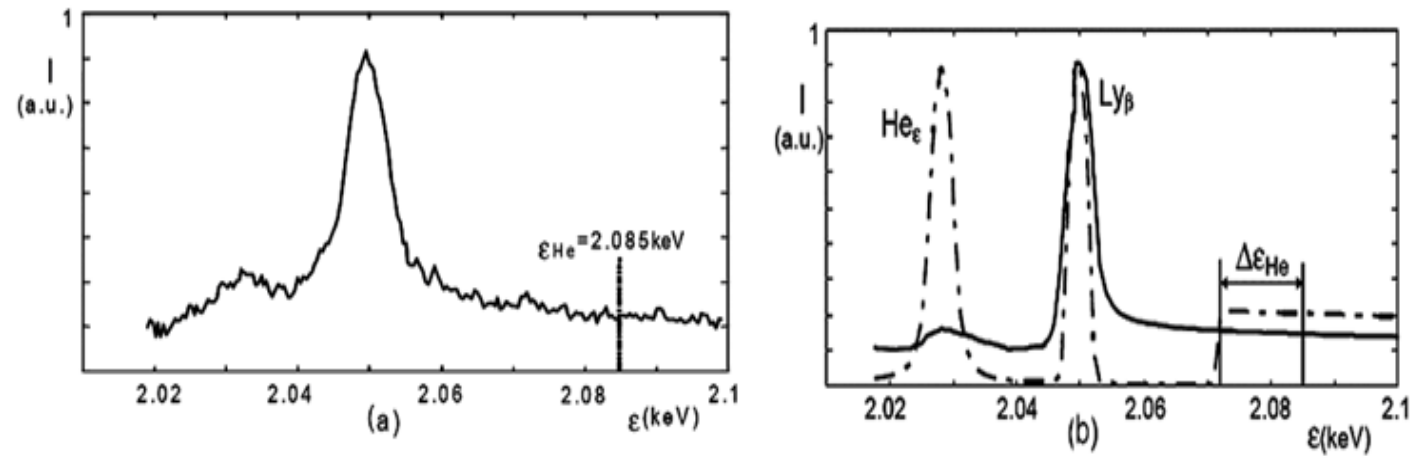

Figure2. Experimental (a) and calculated (b) parts of the micropinch spectrum normalized to the intensity of the line Ly $\beta$, in the ionization threshold of the main state of He-like ions. The solid line in the embodiment b) corresponds to a density of $0.1 \mathrm{~g} / \mathrm{cm}^{3}$, and the dotted line to $0.01 \mathrm{~g} / \mathrm{cm}^{3}$; it was assumed that $T_{e}=$ $0.35 \mathrm{keV}[11]$.

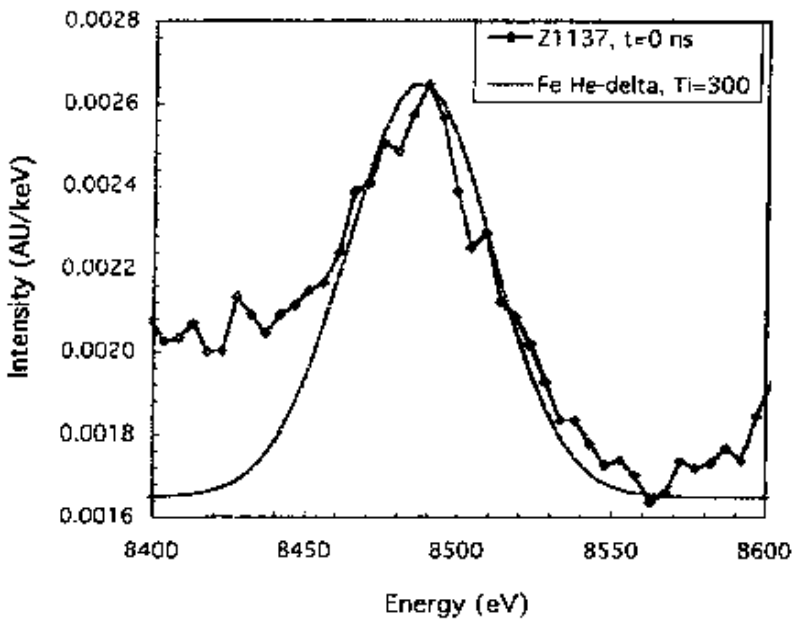

Figure 3. Measured $\mathrm{Fe} \mathrm{He}-\delta$ line at $8.488 \mathrm{keV}$ (broken curve) compared to calculation (smooth curve),[10].

Two groups of researchers independently of each other $[15,16]$ reported that in the X-ray spectra of clusters of galaxies they discovered a new line of radiation with energy of $3.57 \mathrm{keV}$. This emission should come from the hot intergalactic gas that fills the cluster of galaxies, but, unlike other identified lines, this emission line cannot be attributed to any atomic transition. The results of these measurements are shown in Figures 4 and 5. At the same time, the resonance of the spectra of stationary states of electrons in their own gravitational field, and the spectra of multicharge ions can produce not only the registered emission line, but other lines of similar properties[18,19]. It can be expected that in the case of detailed registration of the emission spectra of astrophysical objects, the presence of such lines in the energy range of more than $8 \mathrm{keV}$ can be found. 


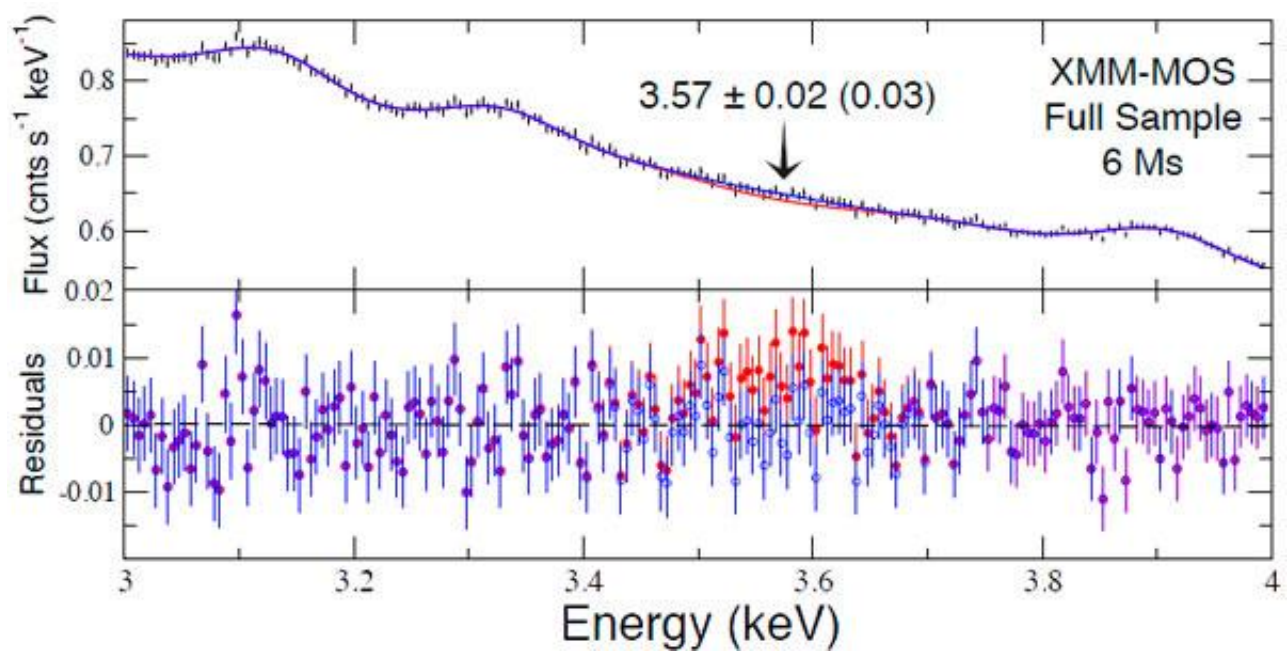

Fig. 4. Above: MOS camera spectrum in the range of 3 to $4 \mathrm{keV}$ of the XMM-Newton observatory. Individual bars are the results of observations with inaccuracy, the red curve is the best reproduction of the spectrum, taking into account only the known ion emission lines, the blue curve is the result of an additive of another previously unknown emission line. Below: the deviation of the observations from the red and blue curves. [15]

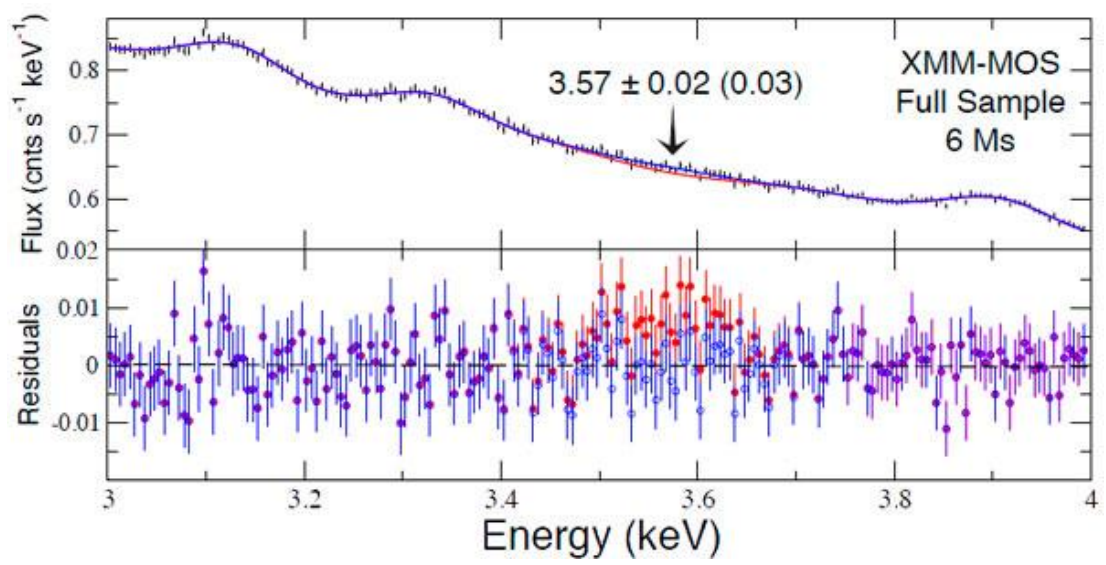

Fig. 5.The X-ray spectrum of the central part of the Andromeda nebula according to the results of MOS-camera monitoring of the XMM-Newton observatory.Left: the entire range from 1 to $8 \mathrm{keV}$, at the right region of 3 to $4 \mathrm{keV}$. Designations are the same as in Fig. 9. [16]

The energy spectrum of the electron in its own gravitational field (Figure 6) and the energy spectra of multielectron atoms (Figure 7,8) are such that there is a resonance of these spectra. As obvious, the consequence of such resonant interaction is appearance, including new lines, of electromagnetic transitions not associated with atomic transitions.

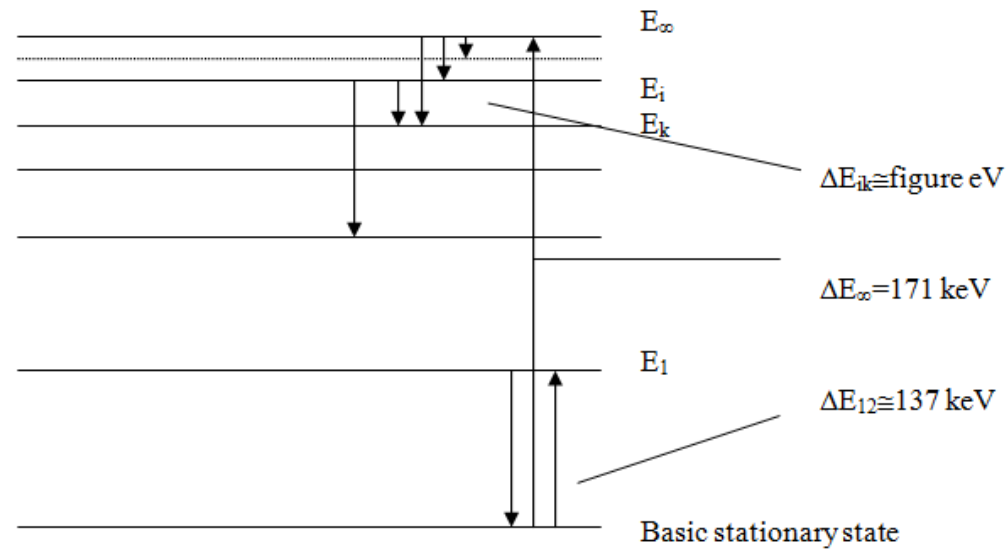

Figure 6.Transitions to stationary states of electrons in proper gravitational field 


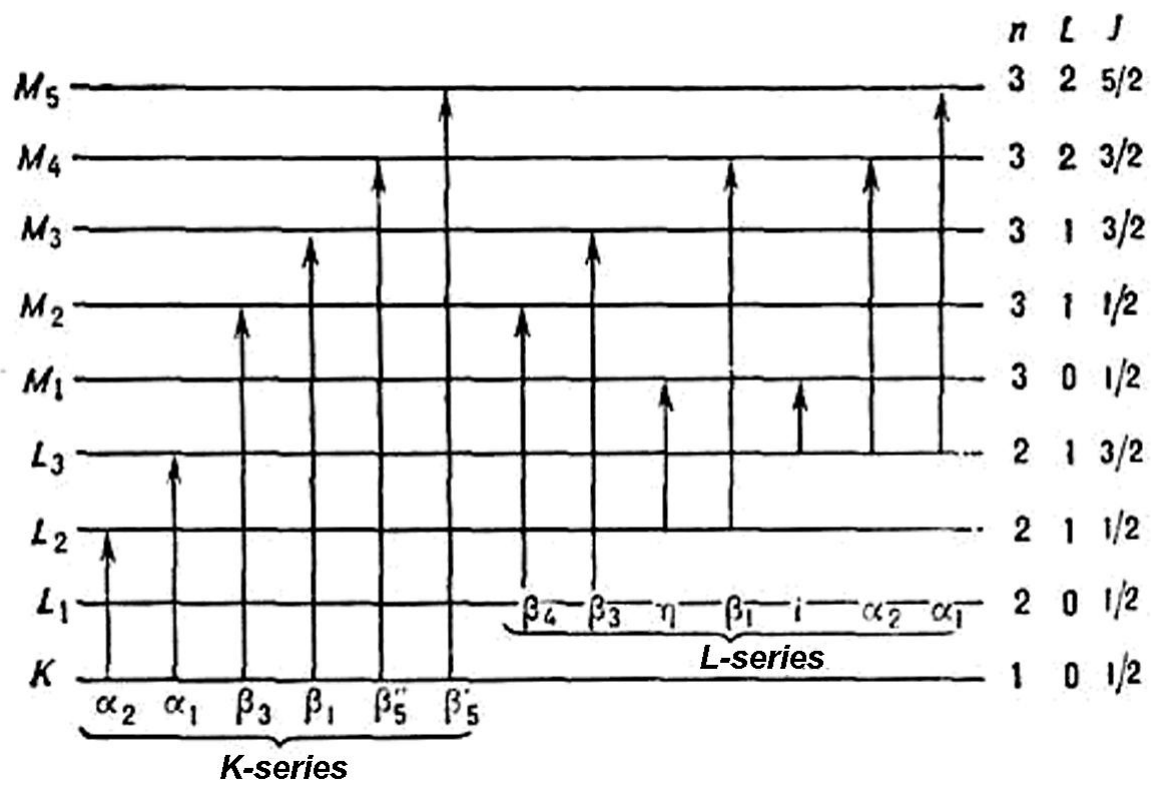

Figure7. Scheme of K-, L- and M-levels of energy of the atom, and the main lines of K- and L- series; $\mathrm{n}, 1, j$ are the principal, the orbital and the inner quantum numbers of energy levels $K, \mathrm{~L}_{1}, \mathrm{~L}_{2}$ etc. The energies of photons of the main lines reach units and scores of $\mathrm{keV}$.

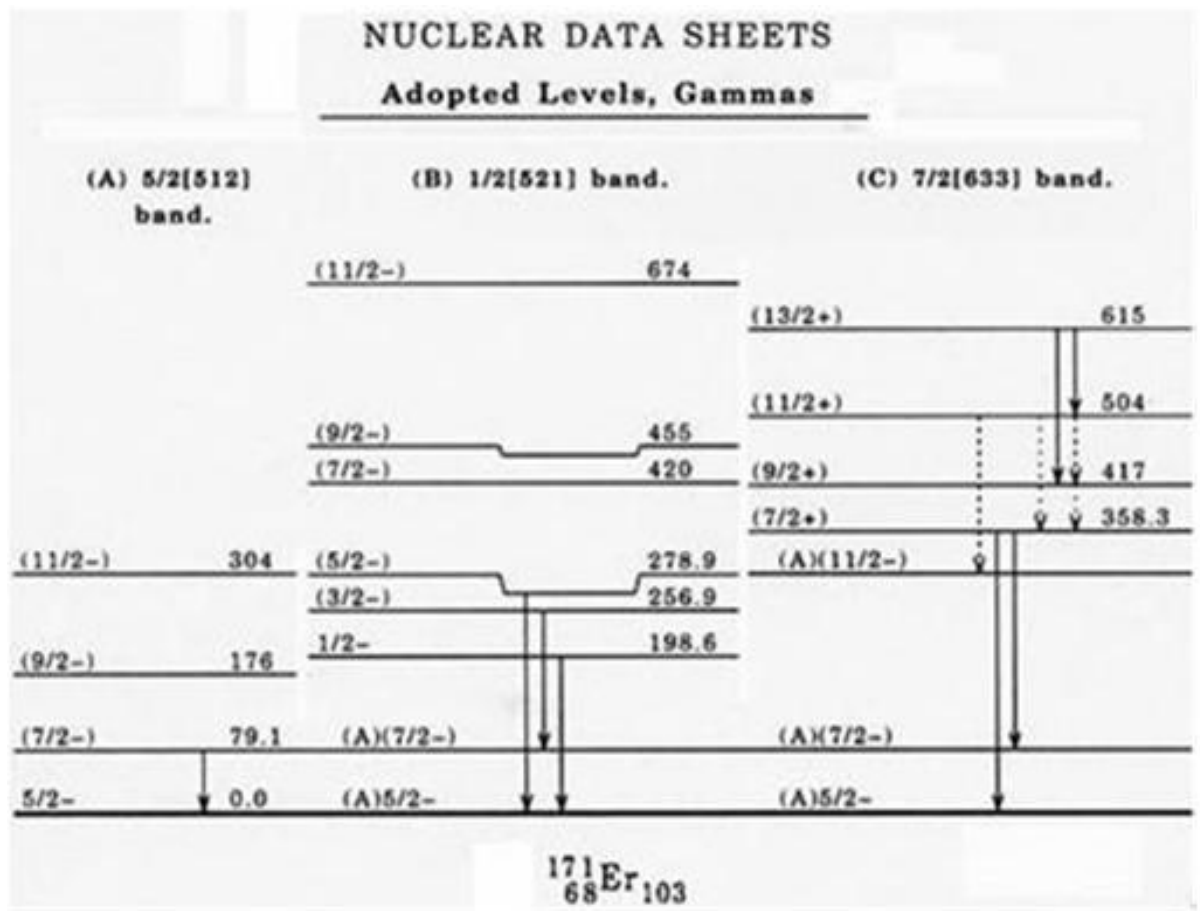

Figure 8.Regular rotational bands in the nucleus of 171Er. Lower rotational energy levels of nuclei are apart from the main one by scores and hundreds of $\mathrm{keV}$.

\section{The states of plasma in the emitting gravitational field}

For the above-indicated energies of transitions over stationary states in the own field and the energy level widths, the sole object in which gravitational emission can be realized as a mass phenomenon will be, as follows from the estimates given below, a dense high-temperature plasma.

Using the Born approximation for the emission cross-section, we can write down the expression for the electromagnetic per unit of volume per unit of time as

$\mathbf{Q}_{\mathrm{e}}=\frac{32}{3} \quad \frac{z^{2} r_{0}{ }^{2}}{137} m c^{2} n_{e} n_{i} \frac{\sqrt{2 k} T_{e}}{\pi m}=0.17 \times 10^{-39} z^{2} n_{e} n_{i} \sqrt{T_{e}}$, 
where $T_{e}, k, n_{i}, n_{e}, m, z, r_{o}$ are the electron temperature, Boltzmann's constant, the concentration of the ionic and electronic components, the electron mass, the serial number of the ionic component, the classical electron radius, respectively.

Replacing $r_{o}$ by $r_{g}=2 \mathrm{~K} \mathrm{~m} / \mathrm{s}^{2}$ (which corresponds to replacing the electric charge e by the gravitational charge $\mathrm{m} \sqrt{\mathrm{K}}$ ), we can use for the gravitational emission the relation $\mathrm{Q}_{\mathrm{g}}=0.16 \mathrm{Q}_{\mathrm{e}}$.

From (16) it follows that in a dense high-temperature plasma with parameters $\mathrm{n}_{\mathrm{e}}=\mathrm{n}_{\mathrm{i}}=10^{23} \mathrm{~m}^{-3}, \mathrm{~T}_{\mathrm{e}}=$ $10^{7} \mathrm{~K}$, the specific power of the electromagnetic emission is equal to $\approx 0.5310^{10} \mathrm{~J} / \mathrm{m}^{3} \mathrm{~s}$, and the specific power of the gravitational emission is $0.8610^{9} \mathrm{~J} / \mathrm{m}^{3} \mathrm{~s}$. These values of the plasma parameters, apparently, can be adopted as guide threshold values of an appreciable gravitational emission level, because the relative proportion of the electrons whose energy on the order of the energy of transitions in the own gravitational field, diminishes in accordance with the Maxwellian distribution exponent as $\mathrm{T}_{\mathrm{e}}$ decreases.

The presence of cascade transitions from the upper excited levels to the lower ones will lead to that the electrons, becoming excited in the energy region above $100 \mathrm{keV}$, will be emitted, mainly, in the eV region, i.e., energy transfer along the spectrum to the low-frequency region will take place. Such energy transfer mechanism can take place only in quenching spontaneous emission from the lower electron energy levels in the own gravitational field, which rules out emission with quantum energy in the keV region. A detailed description of the mechanism of energy transfer along the spectrum will hereafter give its precise numerical characteristics. Nevertheless, undoubtedly, the very fact of its existence, conditioned by the banded character of the spectrum of the gravitational bremsstrahlung, can be asserted. The low-frequency character of the gravitational bremsstrahlung spectrum will lead to its amplification in plasma by virtue of the locking condition $\omega_{g} \leq 0.5 \sqrt{10^{3} n_{e}}$ being fulfilled.

From the standpoint of practical realization of the states of a high-temperature plasma compressed by the emitted gravitational field, two circumstances are of importance. First.Plasma must comprise two components, with multiply charged ions added to hydrogen, these ions being necessary for quenching spontaneous emission of electrons from the ground energy levels in the own gravitational field. For this purpose it is necessary to have ions with the energy levels of electrons close to the energy levels of free excited electrons. Quenching of the lower excited states of the electrons will be particularly effective in the presence of a resonance between the energy of excited electron and the energy of electron excitation in the ion (in the limit, most favorable case - ionization energy). An increase of $\mathrm{z}$ increases also the specific power of the gravitational bremsstrahlung, so that on the condition $\omega_{g} \leq 0.5 \sqrt{10^{3} n_{e}}$ being fulfilled, the equality of the gas-kinetic pressure and the radiation pressure

$$
\mathrm{k}\left(\mathrm{n}_{\mathrm{e}} \mathrm{T}_{\mathrm{e}}+\mathrm{n}_{\mathrm{i}} \mathrm{T}_{\mathrm{i}}\right)=0.16\left(0.1710^{-39} \mathrm{z}^{2} \mathrm{n}_{\mathrm{e}} \mathrm{n}_{\mathrm{i}} \sqrt{\mathrm{T}_{\mathrm{e}}}\right) \Delta \mathrm{t}
$$

will take place at $\Delta t=\left(10^{-6}-10^{-7}\right) \mathrm{s}$ for the permissible parameter values of compressed plasma $\mathrm{n}_{\mathrm{e}}=(1+\mathrm{a}) \mathrm{n}_{\mathrm{i}}$ $=\left(10^{25}-10^{26}\right) \mathrm{m}^{-3}, \mathrm{a}>2, \mathrm{~T}_{\mathrm{e}} \approx \mathrm{T}_{\mathrm{e}}=10^{8} \mathrm{~K}, \mathrm{z}>10$.

Second. The necessity of plasma ejection from the region of the magnetic field with the tentative parameters $n_{e}=\left(10^{23}-10^{24}\right) \mathrm{m}^{-3}, T_{e}=\left(10^{7}-10^{8}\right) \mathrm{K}$ with subsequent energy pumping from the magnetic field region.

\section{Structure of the installation and working compounds}

In the offered method of forming dense-high temperature plasma steady states for nuclear fusion a new fundamental concept is used, namely retaining plasma by radiated gravitational field as radiation of the same kind as electromagnetic:

Forming and accelerating binary plasma with multivalent ions by accelerating magnetic field in a pulse highcurrent discharge.

Injection of binary plasma from the space of the accelerating magnetic field:

Exciting stationary states of an electron in its own gravitational field in the range of energy up to 171 $\mathrm{keV}$ with following radiation under the condition of quenching lower excited energy levels of ion electron shell of a heavy component (including quenching excited state of electrons directly in nuclei of small sequential number as carbon) when retarding plasma bunch ejected from the space of the accelerating magnetic field. Cascade transitions from the upper levels are realized in the process of gravitational radiation energy transit to long-wave range.

The sequence of the operations is carried out in a two-sectional chamber of MAGO installation (Fig. 9, developed in Experimental Physics Research Institute, Sarov; the structure of the installation is most suitable for the claimed method of forming steady states of the dense high-temperature plasma) with magnetodynamic outflow of plasma and further conversion of the plasma bunch energy (in the process of quenching) in the 
plasma heat energy for securing both further plasma heating and exciting gravitational radiation and its transit into a long-wave part of the spectrum with consequent plasma compression in the condition of radiation blocking and increasing .

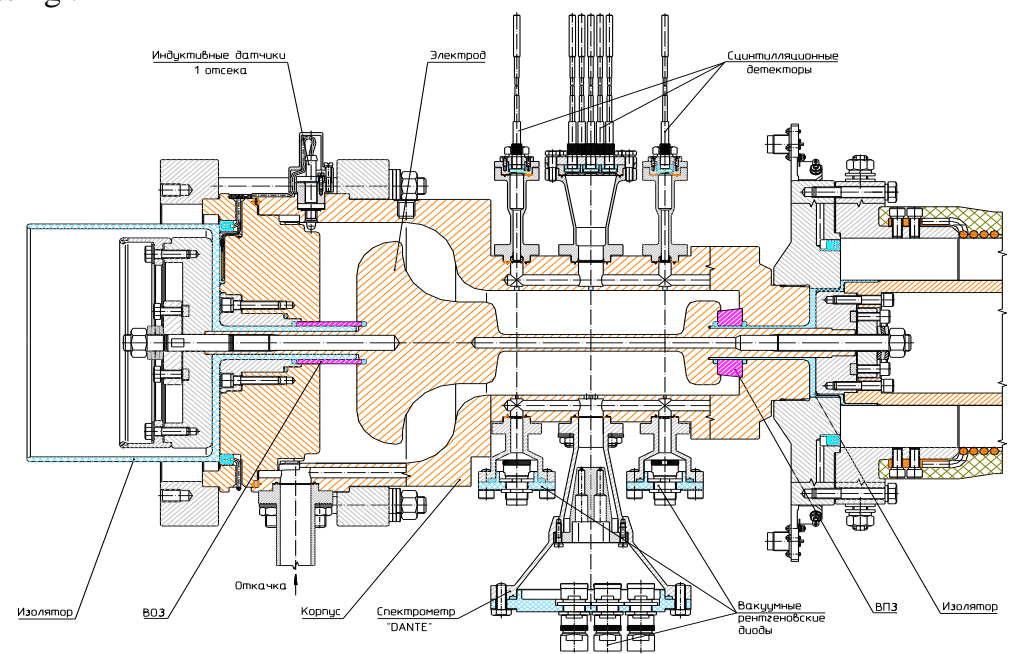

Figure 9. Outline drawing of the discharge chamber (MAGO chamber) and X-ray diagnostic system

Charger-1 Pulsed Power Generator is the most acceptable option to be used in an embodiment of a capacitor discharge device. Assembly of this device has been started in the Aerophysics Research Center (ARC) of the University of Alabama in Huntsville on the basis of the Decade Module Two (DM2) unit. Operating this generator as planned on deuterium-tritium mixture, in practical terms, would be a repetition of the ITER project. At the same time, its usage in the proposed version of the plasma experiment along with MAGO facility would reinforce the project significantly, thus allowing operating both in magnetic explosion mode and in generator with a capacitor battery mode.

Of interest, there are two modes of the installation operations depending on the work gas composition.

1. A composition with hydrogen and (for example) xenon providing for achieving steady states of plasma with consequent realization of thermonuclear reactions for compositions of $(d+t)+$ multi-charge atoms type.

Fusion reaction creating helium generates neutrons

${ }_{1}^{2} \mathrm{H}+{ }_{1}^{3} \mathrm{H} \rightarrow{ }_{2}^{4} \mathrm{He}+{ }_{0}^{1} n+17.6 \mathrm{MeV}$

and was embodied in the well-known Teller-Ulam design with radiation implosion.

An application of the compression-by-the-radiated-gravitational-field design, unlike the Teller-Ulam design, is not limited by the minimum discharge power attendant to the usage of a plutonium nucleus. This means both a feasibility of this fast fusion reaction a steady mode for low-power discharges, and (under certain conditions) a feasibility of explosive energy release without the use of fissile elements (like plutonium and uranium) for highpower discharges.

2. A composition with hydrogen and carbon providing thermonuclear reactions of carbon cycle in plasma steady state mode, including energy pick-up in the form of electromagnetic radiation energy

$\mathrm{CNO}$ cycle is a set of fusion reactions resulting in conversion of hydrogen into helium using carbon as a catalyst.

In a compact notation, this cycle would be written as

${ }^{12} \mathrm{C}(\mathrm{p}, \gamma){ }^{13} \mathrm{~N}\left(\mathrm{e}^{+} \nu\right)^{13} \mathrm{C}\left(\mathrm{p}, \gamma^{14}\right) \mathrm{N}(\mathrm{p}, \gamma)^{15} \mathrm{O}\left(\mathrm{e}^{+} \nu\right)^{15} \mathrm{~N}(\mathrm{p}, \boldsymbol{\alpha}){ }^{12} \mathrm{C}$

The ${ }^{15} \mathrm{~N}(\mathrm{p}, \boldsymbol{\alpha}){ }^{12} \mathrm{C}$ reaction rounds the cycle out. The net result is that four protons turn into in $\alpha$-particle

$-{ }^{4} \mathrm{He}$ nucleus with no neutrons among end products of the cycle. Producing one helium nucleus releases 25

$\mathrm{MeV}$, the produced neutrinos carry away about 5\% more of that energy. A peculiarity of such fusion reaction cycle is that it occurs in natural conditions of astrophysical objects. At the same time, e. g. the design features of MAGO facility allow achieving such a volt-ampere characteristics (VAC) mode that boosts the speed of carbon cycle reactions. An implementation of carbon cycle in the gravitational compression design may possibly become a basic design to form steady states of the thermonuclear plasma, bearing in mind low abundance of hydrogen and lithium isotopes that react with no neutrons being produced either

Above, the spectrum of energies of the electron stationary states in its gravitational field in the simplest form is shown. Accounting for all quantum states provides more detailed spectrum, especially in its upper part. This follows directly from the approximation, in which the above spectrum was obtained. However, it follows already from this approximation that there is possibility of resonance transitions in levels of stationary states of the electromagnetic and gravitational interactions. 
Populations of quantum levels and, consequently, the spectrum characteristics are substantially different in the case of plasma with different content of multiply charged ions. Physically, this is determined by competition between the radiative transition (i.e., spontaneous emission) and nonradioactive transition in the collision of an atom with an electron. Upon excitation of the upper energy levels of electrons in the plasma of multiple-charge ions (when braking on ion nuclei), cascade transitions to lower energy levels will lead to a transfer of gravitational radiation at longer wavelengths followed by blocking and amplification of radiation. In the case when the concentration of multiple-charge ions is negligible, and their range of energy states does not correspond to the possibility of quenching of the lower excited state of electrons, micropinche will take place with its subsequent rapid decay.

\section{Conclusion}

1. If the numerical values of $K \approx 5.1 \times 10^{31} \mathrm{Nm}^{2} \mathrm{~kg}^{-2}$ and $\Lambda=4.4 \times 10^{29} \mathrm{~m}^{-2}$, there is a spectrum of stationary states of the electron in its own gravitational field $(0.511 \mathrm{MeV} \ldots 0.681 \mathrm{MeV})$. The main state is the observed electron rest energy $0.511 \mathrm{MeV}$. This numerical value of $\Lambda$ has important physical meaning: an introduction to the Lagrangian density permanent member, not dependent on the state of the field. This implies the existence of unremovable space-time curvature, not affiliated with any matter, nor with the gravitational field.

2. These steady states are the sources of the gravitational field with constant $G$.

3. Transitions between the stationary states of the electron in its own gravitational field leads to gravitational radiation, which is characterized by a constant $K$, that gravitational radiation is the emission of the same level as electromagnetic (electric charge e, gravitational charge ) $m \sqrt{K}$. The presence of stationary states in the own gravitational field allows the correct calculation of gravitational radiation in the strict quantum approach based on the spectrum of transitions to stationary states already with constant $\mathrm{K}$.

4. Adjusting according to the known mechanisms of broadening does not disclose the broadening of the registered portion of the emission spectrum of the micropinch. It indicates the presence of an additional mechanism of broadening the registered portion of the spectrum of the characteristic radiation due to the contribution of the excited states of electrons in their own gravitational field. The energy spectrum of the electron in its own gravitational field and the energy spectra of multielectron atoms are such that there is a resonance of these spectra. As obvious, the consequence of such resonant interaction is appearance, including new lines, of electromagnetic transitions not associated with atomic transitions

5. In the offered method of forming dense-high temperature plasma steady states for nuclear fusion a new fundamental concept is used, namely retaining plasma by radiated gravitational field as radiation of the same kind as electromagnetic:

Forming and accelerating binary plasma with multivalent ions by accelerating magnetic field in a pulse highcurrent discharge.

Injection of binary plasma from the space of the accelerating magnetic field

The sequence of the operations is carried out in a two-sectional chamber of MAGO installation with magnetodynamic outflow of plasma and further conversion of the plasma bunch energy (in the process of quenching) in the plasma heat energy for securing both further plasma heating and exciting gravitational radiation and its transit into a long-wave part of the spectrum with consequent plasma compression in the condition of radiation blocking and increasing.

\section{References}

[1]. Hilbert, D. Grundlagen der Physik 1 Mitt, math.-nat.kl., 395, Gott. Nachr. (1915).

[2]. Siravam, C. \& Sinha, K. Phys. Rep. 51, 112 (1979).

[3]. Friedmann, A. Zs. Phys. 10, 377 (1922).

[4]. Landau, L.D. \& Lifshitz, E.M. Field Theory (Moscow, Publishing House «Nauka», 1976).

[5]. Warshalovich, D. A. et al. Quantum Theory of Angular Momentum 282-285 (Leningrad, Publishing House «Nauka», 1975).

[6]. Fisenko, S. I. \& Fisenko, I. S. The old and new concepts of physics V6, Number 4, 495-521 (2009).

[7]. Pauli, W. Theory of Relativity (Pergamon Press, 1958).

[8]. Fisenko, S. et al. Phys. Lett. A 148, 8, 9405 (1990).

[9]. Burenkov, O. M. et al. New Configuration of Experiments for MAGO Program. XIV International Conference on Megagauss Magnetic Field Generation and Related Topics (Maui, Havaii, USA), 95-99 (October 14-19, 2012).

[10]. Haines, M. G. et al. Viscous Heating at stagnation in Z-Pinches. PRL 96, 075003-075008 (2006).

[11]. Politov, V.Yu., Potapov, A.V. \& Antonova, L.V. Proceeding of International Conference "V Zababakhin Scientific Proceedings" (1998).

[12]. Fisenko, S. I. \& Fisenko, I. S. The discrete energy spectrum of the gravitational radiation in the relativistic theory of gravitation. International Journal of Theoretical and Applied Physics (IJTAP) Vol. 2 (2), 32-39 (December, 2012).

[13]. Fisenko, S.I. \& Fisenko, I.S. Journal of Modern Physics Vol. 4, Number 4, 481-485 (April, 2013).

[14]. Fissenko, S. \& Fissenko,I. Method of Forming Stable States of Dense High-Temperfature Plasma. PCT Gazette (IPN WO2005/109970 A1), $\square \mathbf{4 6 5 5 3 ~ ( 2 0 0 5 ) . ~}$

[15]. Bulbul, E. et al. Detection of An Unidentified Emission Line in the Stacked X-ray spectrum of Galaxy Clusters. ArXiv http://arxiv.org/abs/1402.2301 (2014). 
[16]. Boyarsky, A., Ruchayskiy, O., Iakubovskyi, D. \& Franse, J. An unidentified line in X-ray spectra of the Andromeda galaxy and Perseus galaxy cluster. arXiv: http://arxiv.org/abs/1402.4119 (2014).

[17]. Fisenko, S.I. \& Fisenko, I.S. Thermonuclear plasma steady states generation. Journal of Applied Mathematics and Physics Volume 2, Number 5 (April, 2014).

[18]. Fisenko, S.I. \& Fisenko, I.S. Is Gravitational Radiation a Radiation of the same level as Electromagnetic Radiation? Journal of Modern Physics Vol. 5, Number 15 1397-1401 (September, 2014).

[19]. Fisenko, S.I.\& Fisenko, I.S. Gravitational radiation as radiation same level of electromagnetic and its generation in pulsed highcurrent discharge. J. Phys.: Conf. Ser. 574 012157; doi:10.1088/1742-6596/574/1/012157 (2015)

[20]. Antognini, A. et al. ArXiv http://de.arxiv.org/abs/1509.03235.

[21]. Fisenko, Stanislav, The problem on stationary states in self gravitational field. Journal of Modern Physics Vol. 7, Number 10 1045-1048 (May, 2016). 